\title{
Spotlight on acupuncture in laboratory animal medicine
}

This article was published in the following Dove Press journal:

Veterinary Medicine: Research and Reports

24 August 2017

Number of times this article has been viewed

\author{
Elizabeth R Magden \\ Department of Veterinary Sciences, \\ Michale E Keeling Center for \\ Comparative Medicine and Research, \\ University of Texas MD Anderson \\ Cancer Center, Bastrop, TX, USA
}

Correspondence: Elizabeth R Magden Department of Veterinary Sciences, Michale E Keeling Center for Comparative Medicine and Research, University of Texas MD Anderson Cancer Center, 650 Cool Water Dr., Bastrop, TX 78602, USA

Tel +5I23327370

Email ermagden@mdanderson.org

\begin{abstract}
Acupuncture has been practiced for thousands of years, although it is only in the past century that science has worked to unravel the mechanisms behind its use. Literature supporting the efficacious use of acupuncture to treat a variety of conditions has been and continues to be published, including the randomized controlled studies we all appreciate when practicing evidence-based medicine. The use of acupuncture in veterinary medicine has paralleled the trends observed in people, with an increasingly common use to remedy specific medical conditions. These conditions are commonly related to neurological dysfunction or orthopedic pain. Although pain relief is the most common use of acupuncture, numerous other conditions have been shown to improve with this therapy. Laboratory animals are also benefiting from acupuncture. Its use is starting to be incorporated into research settings, although there is still further progress to be made in this field. Acupuncture has been shown to improve clinical conditions and quality of life in laboratory animals, and should be considered as a tool to treat laboratory animals with conditions known to benefit from therapy. Here we review the history, mechanisms of action, and use of acupuncture to treat veterinary patients and laboratory animals.
\end{abstract}

Keywords: acupuncture, laboratory animals, nonhuman primates

\section{History and concept}

The origins of acupuncture are abstruse. Some evidence suggests that it has existed as a therapy for thousands of years, with the finding of sharpened stones and bones believed to have served as the earliest needles. ${ }^{1}$ An ancient Chinese textbook, Huangdi Neijing, or The Yellow Emperor's Classic of Internal Medicine is believed to have been written in $100 \mathrm{BCE}$, and describes the use of acupuncture. ${ }^{2}$ In this text, the concept of meridians or channels is well established, although there continues to be some incongruity in the exact anatomical locations of the acupuncture points. ${ }^{2}$ The early treatments involved not only the puncture of the skin, but were also characterized by blood-letting and were quite different from modern-day acupuncture. ${ }^{3}$ Acupuncture has evolved over the past millennia into the practices we use today, the stimulation of tissue, vessels, and nerves without the release of blood.

As the body of literature supporting the use of acupuncture as an effective treatment modality has grown, the use of this integrative therapeutic has increased. There are thousands of studies that support acupuncture as a proven treatment for a wide variety of ailments. Many of these studies evaluate human patients that have shown benefits for disorders such as osteoarthritis, chronic pain, orofacial pain, migraines, gastrointestinal disorders, radiation-induced xerostomia, nausea/vomiting, cardiac 
arrhythmias, hypertension, and stress. ${ }^{4-17}$ Neurological disorders such as stroke and Parkinson's disease have also been shown to benefit from acupuncture treatments. ${ }^{18}$ The National Institutes of Health convened a Consensus Development Conference on Acupuncture in 1997 and issued a statement of support for several conditions in which acupuncture may be a useful adjunct treatment. ${ }^{17}$

Given the vast body of literature supporting the use of acupuncture therapy as a treatment for a defined list of ailments, we should understand the mechanism of action of acupuncture. The benefits of acupuncture are observed when thin, sterile needles are inserted into defined acupuncture points, stimulating physiologic processes through neural signaling. ${ }^{19,20}$ Acupuncture points are primarily located at sites that correspond to neurovascular bundles, blood plexuses, and motor endplate zones. ${ }^{20-22}$ Needles are inserted into these very innervated and vascular acupuncture points, and then rotated gently. The rotation of the needle disrupts the underlying subcutaneous connective tissue, which creates a mechanical signal to the nervous system. ${ }^{23-25}$ The somatic sensory information is carried to the spinal cord and this peripheral afferent signal can lead to one of numerous effects, depending on the site stimulated. As the signal travels to the spinal cord, it can stimulate the dorsal horn which results in the release of endogenous opioids and enhanced analgesia. ${ }^{26-28}$ Internal organ function can be influenced by acupuncture when the visceral neural pathways are stimulated along the bladder meridian, or where the dorsal nerve roots exit the spinal cord. ${ }^{19,29}$ Acupuncture has also been shown to cause vasodilation by increasing the release of vasodilatory peptides such as calcitonin gene-related peptide and vasoactive intestinal polypeptide, which serve to improve circulation and tissue healing. ${ }^{30-32}$

As the nerve signal travels to the brain cortex, it can modulate biomechanical responses such as antinociception to reduce pain, antiemetic to reduce nausea, and prokinetic to promote gastrointestinal mobility. ${ }^{33}$ Acupuncture can also influence hormone regulation, such as testosterone in polycystic ovary syndrome, and may also function to regulate the hypothalamic-pituitary-adrenal axis. ${ }^{34,35}$ Advanced imaging modalities such as functional magnetic resonance imaging and positron emission tomography scans reveal that acupuncture can stimulate different areas of the brain compared to sham therapies, which may indicate possible mechanisms of action that lead to the observed benefits of acupuncture therapy. ${ }^{36-38}$

Electroacupuncture is the application of a low level electrical current to the acupuncture needles, with the goal of a more vigorous stimulation of the acupuncture points. ${ }^{39}$ Some studies have found that electroacupuncture may be more effective than manual acupuncture alone. ${ }^{40}$ Two widely used medical devices, transcutaneous electrical nerve stimulation for pain control and antinausea wrist bands, are in use based on research derived from acupuncture theories. ${ }^{41}$

\section{Acupuncture in veterinary medicine}

The use of acupuncture in treating veterinary companion animals has increased in recent years. It is commonly employed as an adjunct therapy for treating painful conditions, and generally received well by both the patients and clients due to its minimal invasiveness and low risk of adverse events. ${ }^{39}$ In one retrospective study involving over 5,000 mostly small animal patients, the most common conditions which employed acupuncture as an integrative treatment were neurological and orthopedic conditions, followed by internal medicine and oncology cases. ${ }^{42}$ To give a few examples, acupuncture has been used to improve mobility in dogs with musculoskeletal pain, to relieve pain associated with cervical neurological disease in dogs, for respiratory resuscitation of newborn kittens, and for treatment of certain immune-mediated disorders in small animals. ${ }^{43-47}$

Equine veterinarians have also started to use acupuncture with more frequency in their practices. One study found that electroacupuncture provided significant analgesia during equine surgery. ${ }^{48}$ Another study found that acupuncture can assist in equine rehabilitation by promoting tissue healing and muscle strength. ${ }^{49}$ Laryngeal hemiplegia has also been treated using electroacupuncture in horses. ${ }^{50}$

When treating our animal patients, we often have to extrapolate the location of acupuncture points from the associated human points. Human acupuncture points are well defined. ${ }^{51}$ Anatomical landmarks and nerve anatomy can be used to find the associated animal acupuncture points, and the translated points have been researched and published. ${ }^{25,52}$ In animals such as nonhuman primates, translating a human point is fairly straightforward given the similar anatomy. However, more challenges arise when treating dogs, cats, and horses. In these cases, courses on veterinary medical acupuncture can assist the practitioner with point location, as well as a detailed understanding of the nervous system anatomy. On occasion, some points will not be accessible. For example, many of the back points are out of the question when treating a turtle, and the limb acupuncture points are not available if treating a snake. As veterinarians, we need to be flexible and adapt our treatments to our patients. Being adaptable is also key when treating laboratory animals 
to work with the different species and within the defined research parameters.

\section{Acupuncture in laboratory animals}

While the use of acupuncture has expanded to veterinary medicine, and many studies support the efficacious use of acupuncture to treat our companion animals such as dogs, cats, and horses, the use of acupuncture in the laboratory animal medicine field has spread more slowly. This slow acceptance has several root causes. Many laboratory species are rodents, and their small size can make proper acupuncture technique and point location more difficult. Many laboratory animal veterinarians have not received training in acupuncture and may be unaware of the treatment benefits. While the cost of acupuncture needles is low, the time investment is high, and some veterinarians do not have the time to incorporate acupuncture into their treatment plans. There is also some concern raised by investigators that acupuncture could introduce an unknown variable into the study results since laboratory animals are often serving on research studies. Additionally, scientists working with laboratory animals may not believe that acupuncture works, or may not be convinced by the evidence-based research that supports the use of acupuncture to treat a wide variety of health conditions.

Despite all these concerns, there has been progress made in providing acupuncture therapy to laboratory animals. Chimpanzees (Pan troglodytes) with osteoarthritis have been successfully treated with acupuncture. Veterinarians and animal behaviorists trained the chimpanzees to voluntarily present the body part of interest using positive reinforcement training. The veterinarian used dental floss tied around the acupuncture needles to ensure the needles remained with the providers and to protect the animals from a lost needle or unintentional ingestion (Figure 1). ${ }^{53}$ The acupuncture was found to improve mobility in the chimpanzees receiving therapy. ${ }^{53}$ Chimpanzees have also been trained to present their wrists for acupuncture to treat cardiac arrhythmias, using the acupuncture points Pericardium-6 (PC-6) and Heart-7 (HT-7) (Figure 2). This treatment was based on human studies that found an improvement in various types of cardiac arrhythmias with the use of these acupuncture points. ${ }^{54,55}$ In the chimpanzee, cardiac arrhythmias were monitored using an implantable loop recorder, and the frequency of arrhythmias was documented during both pharmacologic and acupuncture treatments. ${ }^{56}$

Other nonhuman primate species have benefited from acupuncture therapy. Owl monkeys (Aotus spp.) have been treated for osteoarthritis and accepted gentle manual restraint

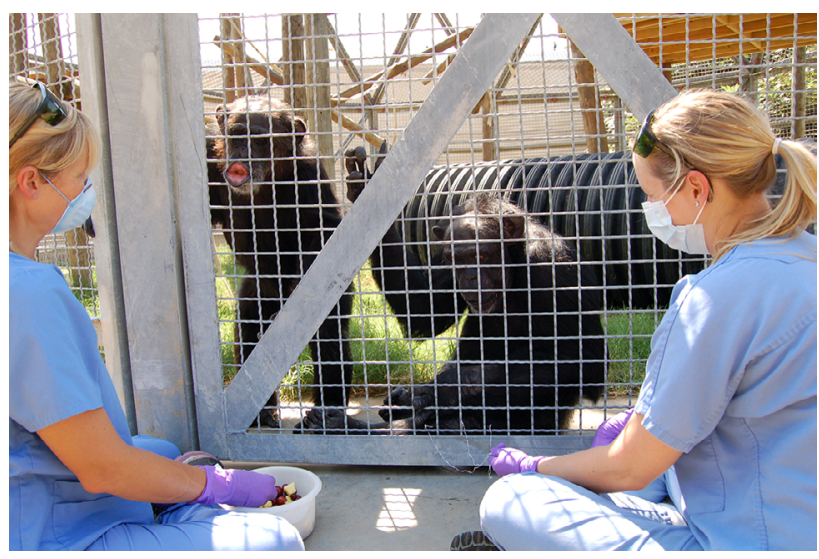

Figure I Chimpanzee (Pan troglodytes) sitting and receiving acupuncture therapy for stifle osteoarthritis at points ST-34, ST-35, and ST-36 with dental floss tied around acupuncture needles to prevent needle loss.

Note: ST refers to specific acupuncture points along the stomach channel.

Abbreviation: ST, Stomach.

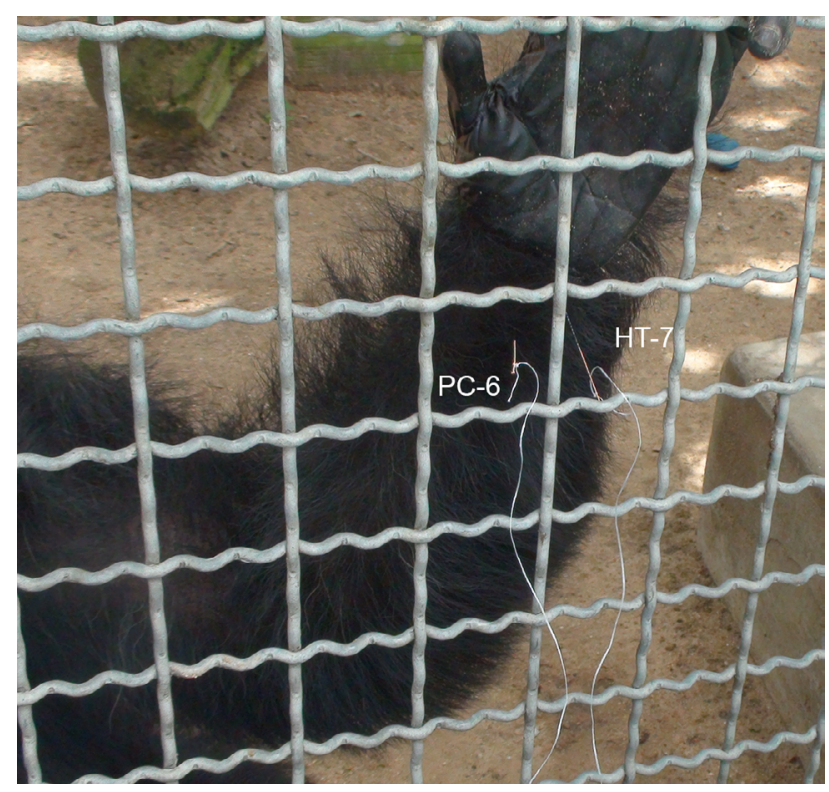

Figure 2 Chimpanzee (Pan troglodytes) receiving acupuncture at the wrist location for points PC-6 and HT-7 for treatment of cardiac arrhythmias.

Abbreviations: PC, pericardium; HT, heart.

throughout the acupuncture treatment (Figure 3). ${ }^{57}$ Once acclimated to the therapy, the owl monkeys would relax and almost fall asleep during the acupuncture treatments. Rhesus macaques (Macaca mulatta) and squirrel monkeys (Saimiri spp.) have also been treated for various conditions such as soft tissue injury and have become habituated to gentle manual restraint for the duration of the acupuncture procedures. ${ }^{57}$

While there are a limited number of studies on treating spontaneous disease in laboratory animals, there are numerous studies that have examined the role of acupuncture in experimental disease processes. Not only can we learn more about potential effective acupuncture treatments to benefit 


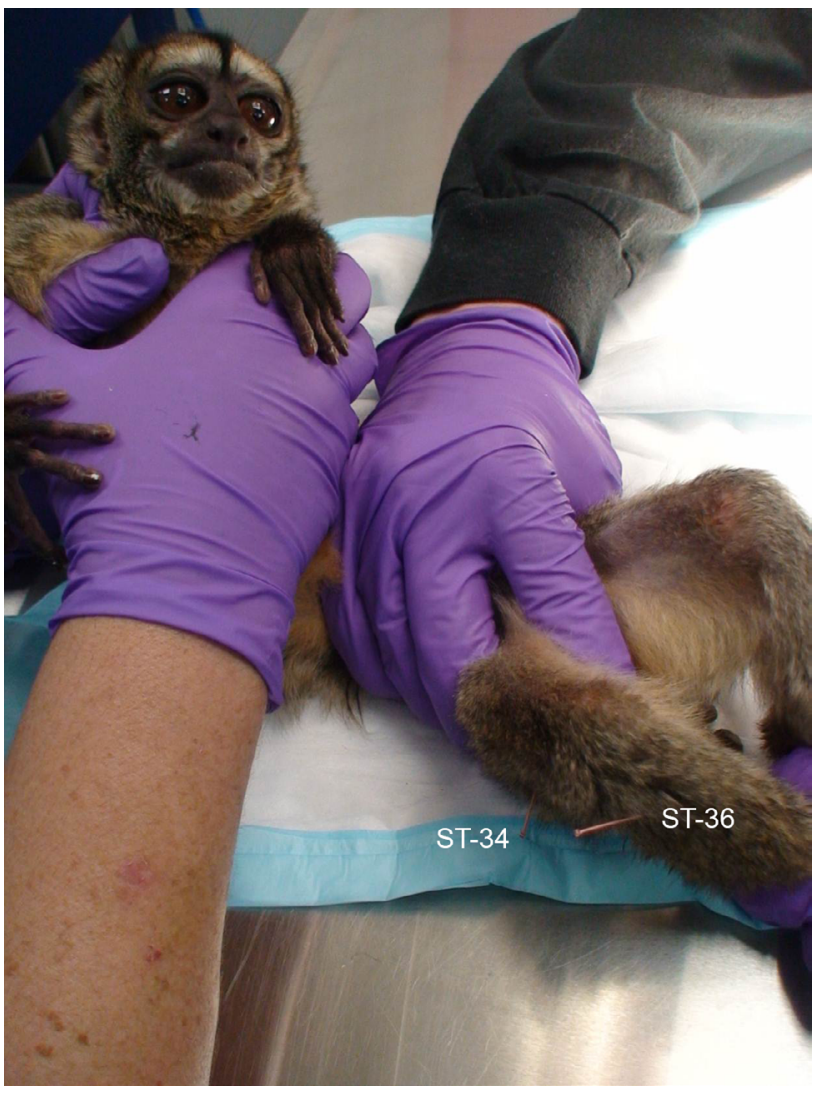

Figure 3 Owl monkey (Aotus spp.) receiving acupuncture for stifle osteoarthritis in points ST-34 and ST-36. Gentle manual restraint is used for the duration of the therapy.

Note: ST refers to specific acupuncture points along the stomach channel. Abbreviation: ST, Stomach.

the animals, we can also learn about potential treatments that can be used to ameliorate human disease processes. Animal studies have been instrumental in determining the effectiveness of acupuncture, as they are not subject to the same placebo effects as human subjects.

Rhesus macaques with Parkinsonian-like symptoms have been trained to accept electroacupuncture treatments, and the impaired monkeys showed improved movement speed with treatment. ${ }^{58}$ Rhesus macaques have also exhibited increased analgesia with acupuncture therapy. Positron emission tomography scans have been utilized to visualize areas in the brain that responded to acupuncture analgesia in the rhesus macaques. ${ }^{59}$ Electroacupuncture has been shown to have neuroprotective effects in nonhuman primate models of cerebral ischemia and stroke. ${ }^{60}$ In support of the analgesic effects of acupuncture, it has also been shown that naloxone can reverse the analgesic effects of acupuncture in nonhuman primates. ${ }^{61}$

In rodents and rabbits, there are numerous studies investigating the benefits of acupuncture. Rats treated with electroacupuncture following cerebral hemorrhage showed improved clinical outcomes. ${ }^{62,63}$ A mouse model of fibromyalgia revealed enhanced pain attenuation with the use of electroacupuncture. ${ }^{64}$ In rabbits undergoing ovariohysterectomies, electroacupuncture was found to provide good analgesia for postoperative pain. ${ }^{65}$

Some laboratory animal veterinarians and researchers may not be aware that many of the studies investigating the mechanisms of action of acupuncture have done so with the contributions of laboratory animals. The analgesia that is often observed with acupuncture can be attributed to the release of endogenous opioid peptides. Levels of enkephalin and $\beta$-endorphins have been shown to increase in serum and spinal samples following acupuncture in rabbits. ${ }^{66}$ The immunomodulatory effects of acupuncture have been examined in rats treated with electroacupuncture stimulation. The study showed an enhanced level of immune cytokines (interferon- $\gamma$ ), supporting the proposed immunomodulatory function of acupuncture therapy. ${ }^{67} \mathrm{~A}$ neurodegeneration study with mice found that electroacupuncture has a time dependent effect on the levels of proinflammatory and anti-inflammatory cytokines ${ }^{68}$ In rats, electroacupuncture has also been shown to stimulate the release of mesenchymal stem cells that act to promote tissue repair, increase levels of anti-inflammatory cytokines, and enhance analgesia. ${ }^{69}$

To maximize the benefits of acupuncture, anesthesia should be avoided when possible due to its suppressive effects on neuronal activity. ${ }^{12}$ The potential adverse effects of acupuncture are low when performed correctly. Acupuncturists should avoid joint spaces, lung fields, and ensure needles do not break off into the tissue. Other risks include minor bleeding or possible infection, although the incidence is very low. The biggest risk when using acupuncture to treat laboratory animals are the potential scientific complications. For example, if there is a study investigating inflammatory disease and the animals cannot receive nonsteroidal anti-inflammatory drugs, it is very likely they also should not receive acupuncture therapy due to the know anti-inflammatory effects of acupuncture. ${ }^{70}$ Researchers and veterinarians can work together to determine if acupuncture is a viable treatment option and ensure this therapy does not become an unwelcome research variable.

\section{Conclusion}

In a science-based field such as laboratory animal medicine, we need to have a basis for treatment of the research animals. In determining if an animal would benefit from acupuncture treatment, the first step is to search the literature and determine if a particular illness or injury has been successfully 
treated in the past by integrating acupuncture into the treatment plan. Many times, the studies are human studies. Nonhuman primates are phylogenetically close to humans, and so it can be reasonably extrapolated that if acupuncture worked to treat a human, it will also likely be successful in treating a nonhuman primate. As we work down the phylogenetic tree to rodents, rabbits, and dogs, we will often encounter a condition that may not have been treated with acupuncture previously in a particular species. We can continue to look toward other closely related species for information on how a laboratory animal may respond, and work on translating the acupuncture points from human to mouse, dog, or pig. Once we successfully (or unsuccessfully) use acupuncture treatments in a laboratory animal, we should publish our results. These publications will form a legitimate basis for future veterinarians and animal technicians to consider integrating acupuncture at their home facilities. Given the low risk of adverse effects and observed benefits for animal health and well-being, acupuncture should be considered as a potential tool in all veterinarian treatment strategies.

\section{Disclosure}

The author reports no conflicts of interest in this work.

\section{References}

1. Ma K. The roots and development of Chinese acupuncture: from prehistory to early 20th century. Acupunct Med. 1992;10:92-99.

2. White A, Ernst E. A brief history of acupuncture. Rheumatology. 2004;43(5):662-663.

3. Epler DC, Jr. Bloodletting in early Chinese medicine and its relation to the origin of acupuncture. Bull His Med. 1980;54(3):337-367.

4. Chiu YJ, Chi A, Reid IA. Cardiovascular and endocrine effects of acupuncture in hypertensive patients. Clin Exp Hypertens. 1997;19(7):1047-1063.

5. Goddard G. Short term pain reduction with acupuncture treatment for chronic orofacial pain patients. Med Sci Monit. 2005;11(2):Cr71-Cr74.

6. Zhang Y, Bao F, Wang Y, Wu Z. Influence of acupuncture in treatment of knee osteoarthritis and cartilage repairing. Am J Transl Res. 2016;8(9):3995-4002.

7. Lombardi F, Belletti S, Battezzati PM, Lomuscio A. Acupuncture for paroxysmal and persistent atrial fibrillation: An effective nonpharmacological tool? World J Cardiol. 2012;4(3):60-65.

8. Mavrommatis CI, Argyra E, Vadalouka A, Vasilakos DG. Acupuncture as an adjunctive therapy to pharmacological treatment in patients with chronic pain due to osteoarthritis of the knee: a 3-armed, randomized, placebo-controlled trial. Pain. 2012;153(8):1720-1726.

9. Middlekauff HR, Yu JL, Hui K. Acupuncture effects on reflex responses to mental stress in humans. Am J Physiol. 2001;280(5):R1462-R1468.

10. Ouyang H, Chen JD. Review article: therapeutic roles of acupuncture in functional gastrointestinal disorders. Aliment Pharmacol Ther. 2004;20(8):831-841.

11. Cheng L, Li P, Tjen-A-Looi SC, Longhurst JC. What do we understand from clinical and mechanistic studies on acupuncture treatment for hypertension? Chinese Med. 2015;10:36.

12. Zhao XF, Hu HT, Li JS, et al. Is acupuncture effective for hypertension? A systematic review and meta-analysis. PLoS One. 2015;10(7):e0127019.
13. MacPherson H, Vertosick EA, Foster NE, et al. The persistence of the effects of acupuncture after a course of treatment: a meta-analysis of patients with chronic pain. Pain. 2017;158(5):784-793.

14. Blom M, Dawidson I, Fernberg JO, Johnson G, Angmar-Mansson B. Acupuncture treatment of patients with radiation-induced xerostomia. Eur J Cancer. 1996;32(3):182-190.

15. Scallan EM, Simon BT. The effects of acupuncture point Pericardium 6 on hydromorphone-induced nausea and vomiting in healthy dogs. Vet Anaesth Analg. 2016;43(5):495-501.

16. Yang A, Wu HM, Tang JL, Xu L, Yang M, Liu GJ. Acupuncture for stroke rehabilitation. Cochr Database Syst Rev. 2016;8:Cd004131.

17. Acupuncture NINCDPo. Acupuncture. JAMA. 1998;280(17):1518-1524.

18. Lam YC, Kum WF, Durairajan SS, et al. Efficacy and safety of acupuncture for idiopathic Parkinson's disease: a systematic review. JAltern Comp Med. 2008;14(6):663-671.

19. Robinson NG. Veterinary acupuncture, an ancient tradition for modern times. Altern Comp Therap. 2007;13(5):259-265.

20. Robinson NG. Making sense of the metaphor: how acupuncture works neurophysiologically. J Equine Veterin Sci. 29(8):642-644.

21. Dung $\mathrm{H}$. Anatomical features contributing to the formation of acupuncture points. Am J Acupunct.1984;12:139-143.

22. Ogay V, Min F, Kim K, et al. Observation of coiled blood plexus in rat skin with diffusive light illumination. J Acupunct Meridian Stud. 2009;2(1):56-65.

23. Langevin HM, Yandow JA. Relationship of acupuncture points and meridians to connective tissue planes. Anat Rec. 2002;269(6): 257-265.

24. Fox JR, Gray W, Koptiuch C, Badger GJ, Langevin HM. Anisotropic tissue motion induced by acupuncture needling along intermuscular connective tissue planes. J Altern Complement Med. 2014;20(4):290-294.

25. Robinson NG. One medicine, One acupuncture. Animals. 2012;2(3): 395-414.

26. Uchida Y, Nishigori A, Takeda D, et al. Electroacupuncture induces the expression of Fos in rat dorsal horn via capsaicin-insensitive afferents. Brain Res. 2003;978(1-2):136-140.

27. Zhao ZQ. Neural mechanism underlying acupuncture analgesia. Prog Neurobiol. 2008;85(4):355-375.

28. Kawakita K, Okada K. Acupuncture therapy: mechanism of action, efficacy, and safety: a potential intervention for psychogenic disorders? Biopsych Med. 2014;8:4.

29. Yin J, Chen JD. Gastrointestinal motility disorders and acupuncture. Auton Neurosci. 2010;157(1-2):31-37.

30. Lundeberg T. Peripheral effects of sensory nerve stimulation (acupuncture) in inflammation and ischemia. Scand J Rehabil Med. 1993;29:61-86.

31. Dawidson I, Angmar-Mansson B, Blom M, Theodorsson E, Lundeberg T. Sensory stimulation (acupuncture) increases the release of calcitonin gene-related peptide in the saliva of xerostomia sufferers. Neuropeptides. 1999;33(3):244-250.

32. Dawidson I, Angmar-Mansson B, Blom M, Theodorsson E, Lundeberg T. Sensory stimulation (acupuncture) increases the release of vasoactive intestinal polypeptide in the saliva of xerostomia sufferers. Neuropeptides. 1998;32(6):543-548.

33. Takahashi T. Mechanism of acupuncture on neuromodulation in the gut--a review. Neuromodulation. 2011;14(1):8-12; discussion 12.

34. Jedel E, Labrie F, Oden A, et al. Impact of electro-acupuncture and physical exercise on hyperandrogenism and oligo/amenorrhea in women with polycystic ovary syndrome: a randomized controlled trial. Am J Physiol Endocrinol Metab. 2011;300(1):E37-E45.

35. Wei Y, Dong M, Zhong L, et al. Regulation of hypothalamic-pituitaryadrenal axis activity and immunologic function contributed to the anti-inflammatory effect of acupuncture in the OVA-induced murine asthma model. Neurosci Lett. 2017;636:177-183.

36. Lu Y, Huang Y, Tang C, et al. Brain areas involved in the acupuncture treatment of AD model rats: a PET study. BMC Complem Altern Med. 2014;14:178. 
37. Yang J, Zeng F, Feng Y, et al. A PET-CT study on the specificity of acupoints through acupuncture treatment in migraine patients. $B M C$ Complem Altern Med. 2012;12:123.

38. Lai X, Zhang G, Huang Y, et al. A cerebral functional imaging study by positron emission tomography in healthy volunteers receiving true or sham acupuncture needling. Neurosci Lett. 2009;452(2):194-199.

39. Fry LM, Neary SM, Sharrock J, Rychel JK. Acupuncture for analgesia in veterinary medicine. Topics Companion Animal Med.2014;29(2):35-42.

40. Ulett GA, Han S, Han JS. Electroacupuncture: mechanisms and clinical application. Biol Psychiat. 1998;44(2):129-138.

41. MacPherson H, Hammerschlag R, Coeytaux RR, et al. Unanticipated insights into biomedicine from the study of acupuncture. J Alter Comp Med. 2016;22(2):101-107.

42. Shmalberg J, Memon MA. A retrospective analysis of 5,195 patient treatment sessions in an integrative veterinary medicine service: patient characteristics, presenting complaints, and therapeutic interventions. Veter Med Int. 2015;2015:983621.

43. Lane DM, Hill SA. Effectiveness of combined acupuncture and manual therapy relative to no treatment for canine musculoskeletal pain. Can Vet J. 2016;57(4):407-414.

44. Liu CM, Chang FC, Lin CT. Retrospective study of the clinical effects of acupuncture on cervical neurological diseases in dogs. J Veter Sci. 2016;17(3):337-345.

45. Skarda RT. Anesthesia case of the month. Dystocia, cesarean section and acupuncture resuscitation of newborn kittens. J Am Veter Med Assoc. 1999;214(1):37-39.

46. Rogers PA, Schoen AM, Limehouse J. Acupuncture for immunemediated disorders. Literature review and clinical applications. Prob Veter Med. 1992;4(1):162-193.

47. Janssens L, Altman S, Rogers PA. Respiratory and cardiac arrest under general anaesthesia: treatment by acupuncture of the nasal philtrum. Veter Rec. 1979;105(12):273-276.

48. Sheta E, Ragab S, Farghali H, El-Sherif A. Successful practice of electroacupuncture analgesia in equine surgery. $J$ Acupunct Merid Stud. 2015;8(1):30-39.

49. le Jeune S, Henneman K, May K. Acupuncture and equine rehabilitation. The veterinary clinics of North America. Equine Prac. 2016;32(1):73-85.

50. Kim MS, Xie H. Use of electroacupuncture to treat laryngeal hemiplegia in horses. Veter Rec. 2009;165(20):602-604.

51. White A. Reference charts: points and innervation A2. In: Cummings M, Filshie J, editors. An Introduction to Western Medical Acupuncture. Edinburgh: Churchill Livingstone; 2008:185-218.

52. Lancaster LS, Bowker RM. Acupuncture points of the horse's distal thoracic limb: a neuroanatomic approach to the transposition of traditional points. Animals. 2012;2(3):455-471.

53. Magden ER, Haller RL, Thiele EJ, Buchl SJ, Lambeth SP, Schapiro SJ. Acupuncture as an adjunct therapy for osteoarthritis in chimpanzees (Pan troglodytes). J Am Assoc Lab Animal Sci. 2013;52(4):475-480.

54. VanWormer AM, Lindquist R, Sendelbach SE. The effects of acupuncture on cardiac arrhythmias: a literature review. Heart Lung. 2008;37(6):425-431.
55. Liu J, Li SN, Liu L, et al. Conventional acupuncture for cardiac arrhythmia: A systematic review of randomized controlled trials. Chinese $J$ Integr Med. Epub 2017 Apr 22.

56. Magden ER, Sleeper MM, Buchl SJ, Jones RA, Thiele EJ, Wilkerson GK. Use of an implantable loop recorder in a Chimpanzee (Pan troglodytes) to monitor cardiac arrhythmias and assess the effects of acupuncture and laser therapy. Comp Med. 2016;66(1):52-58.

57. Magden ER, Mansfield KG, Simmons JH, Abee CR. Nonhuman primates A2 - fox, James G. In: Anderson LC, Otto GM, Pritchett-Corning KR, Whary MT, editors. Laboratory Animal Medicine. 3rd ed. Boston, MA: Academic Press; 2015:771-930.

58. Zhao F, Fan X, Grondin R, et al. Improved methods for electroacupuncture and electromyographic recordings in normal and parkinsonian rhesus monkeys. J Neurosci Methods. 2010;192(2):199-206.

59. Maenaka T, Tano K, Nakanishi S, Tsukada H, Ishida T. Positron emission tomography analysis of the analgesic effects of acupuncture in rhesus monkeys. Am J Chinese Med. 2006;34(5):787-801.

60. Gao H, Guo J, Zhao P, Cheng J. The neuroprotective effects of electroacupuncture on focal cerebral ischemia in monkey. Acupunct ElectroTherap Res. 2002;27(1):45-57.

61. Ha H, Tan EC, Fukunaga H, Aochi O. Naloxone reversal of acupuncture analgesia in the monkey. Exp Neurol. 1981;73(1):298-303.

62. Li Z, Zheng X, Li P, Itoua ES, Moukassa D, Ndinga Andely F. Effects of acupuncture on mRNA levels of apoptotic factors in perihematomal brain tissue during the acute phase of cerebral hemorrhage. Med Sci Monit. 2017;23:1522-1532.

63. Zhu Y, Deng L, Tang H, et al. Electroacupuncture improves neurobehavioral function and brain injury in rat model of intracerebral hemorrhage. Brain Res Bull. Epub 2017 Apr 7.

64. Yen LT, Hsieh CL, Hsu HC, Lin YW. Targeting ASIC3 for relieving mice fibromyalgia pain: roles of electroacupuncture, opioid, and adenosine. Sci Rep. 2017;7:46663.

65. Parmen V. Electroacupuncture analgesia in a rabbit ovariohysterectomy. J Acupunct Merid Stud. 2014;7(1):15-24.

66. Hsieh YL, Hong CZ, Liu SY, Chou LW, Yang CC. Acupuncture at distant myofascial trigger spots enhances endogenous opioids in rabbits: a possible mechanism for managing myofascial pain. Acupunct Med. 2016;34(4):302-309.

67. Chen L, Xu A, Yin N, et al. Enhancement of immune cytokines and splenic CD4+ T cells by electroacupuncture at ST36 acupoint of SD rats. PLoS One 2017;12(4):e0175568

68. He J, Liao T, Zhong GX, et al. Alzheimer`s disease-like early-phase brain pathogenesis: Self-curing amelioration of neurodegeneration from pro-inflammatory 'wounding' to anti-inflammatory 'healing'. Curr Alzheimer Res. Epub 2017 Apr 17.

69. Salazar TE, Richardson MR, Beli E, et al. Electroacupuncture promotes central nervous system-dependent release of mesenchymal stem cells. Stem Cells. 2017;35(5):1303-1315.

70. Zijlstra FJ, van den Berg-de Lange I, Huygen F, Klein J. Anti-inflammatory actions of acupuncture. Mediat Inflamm. 2003;12(2):59-69.
Veterinary Medicine: Research and Reports

\section{Publish your work in this journal}

Veterinary Medicine: Research and Reports is an international, peer-reviewed, open access journal publishing original research, case reports, editorials, reviews and commentaries on all areas of veterinary medicine. The manuscript management system is completely online and includes a very quick and fair peer-review system.

\section{Dovepress}

Visit http://www.dovepress.com/testimonials.php to read real quotes from published authors. 